\title{
On the Reduction of the Regular Representation of the Poincaré Group
}

\author{
G. RIDEAU \\ Institut Poincaré, Paris \\ Received February 14, 1966
}

Abstract. The decomposition of the regular representation of the Poincare group into irreducible representations is given.

\section{I}

We denote by $(a, \mathcal{A})$ any element of the Poincaré group $\mathscr{P}$, where $a$ is a 4-Translation and $\Lambda$ an element of the Lorentz group $G$. In the following, we shall not distinguish between $G$ and its universal covering $S L(2, C)$. The multiplication law in $\mathscr{P}$ is given by:

$$
\left(a_{1}, \Lambda_{1}\right)\left(a_{2}, \Lambda_{2}\right)=\left(a_{1}+\Lambda_{1} a_{2}, \Lambda_{1} \Lambda_{2}\right) .
$$

We consider the Hilbert space $\mathscr{H}$, the elements of which are functions with square modulus integrable with respect to Haar measure. The mapping

$$
f(a, \Lambda) \stackrel{\left(a_{0}, \Lambda_{0}\right)}{\longrightarrow} f\left(a+\Lambda a_{0}, \Lambda \Lambda_{0}\right)
$$

defines a unitary representation of $\mathscr{P}$, the so-called right regular representation. In this work, we shall explicitly decompose this representation into irreducible components.

We set:

$$
\hat{f}(\hat{a}, \Lambda)=\int f(a, \Lambda) e^{-i \Lambda^{-1} a \cdot \hat{a}} d a
$$

where $a \cdot b$ is the Lorentzian scalar product. Now:

$$
\begin{gathered}
f(a, \Lambda)=\frac{1}{(2 \pi)^{4}} \int \hat{f}(\hat{a}, \Lambda) e^{i \Lambda^{-1} a \cdot \hat{a}} d \hat{a} \\
\left.\int\left|f(a, \Lambda)^{2} d a d \Lambda=\frac{1}{(2 \pi)^{4}} \int\right| \hat{f}(\hat{a}, \Lambda)\right|^{2} d \hat{a} d \Lambda .
\end{gathered}
$$

Therefore, equation (3) defines an isometric mapping of $\mathscr{H}$ into $\hat{\mathscr{H}}$, Hilbert space, the elements of which are functions with square modulus integrable with respect to measure $d \hat{a} d \Lambda$.

Transformation (2) induces in $\hat{\mathscr{H}}$ :

$$
\hat{f}(\hat{a}, \Lambda) \stackrel{\left(a_{0}, \Lambda_{0}\right)}{\longrightarrow} e^{i a_{0} \cdot \hat{a}} \hat{f}\left(\Lambda_{0}^{-1} \hat{a}, \Lambda \Lambda_{0}\right) .
$$


Let, generally, $\Omega_{m}$ be the hyperboloid:

$$
\hat{a} \cdot \hat{a}=m^{2}
$$

and, if $m^{2}>0$, let $\Omega_{m}^{+}, \Omega_{m}^{-}$be superior and inferior sheets of $\Omega_{m}$.

We set:

$$
\left.\begin{array}{lll}
\hat{f}_{m^{2}}(\hat{a}, \Lambda)=\hat{f}(\hat{a}, \Lambda) & \text { for } \quad \hat{a} \in \Omega_{m}, m^{2}<0 \\
\hat{f}_{m^{2}}^{ \pm}(a, \Lambda)=\hat{f}(\hat{a}, \Lambda) & \text { for } \quad \hat{a} \in \Omega_{m}^{ \pm}, m^{2}>0 .
\end{array}\right\}
$$

Now, taking into account equation (6), we have:

and, obviously:

$$
\left.\begin{array}{l}
\hat{f}_{m^{2}}(\hat{a}, \Lambda) \stackrel{\left(a_{0} \cdot \Lambda_{0}\right)}{\longrightarrow} e^{i a_{0} \cdot \hat{a}} \hat{f}_{m^{2}}\left(\Lambda_{0}^{-1} \hat{a}, \Lambda \Lambda_{0}\right) \\
\hat{f}_{m^{2}}^{ \pm}(\hat{a}, \Lambda) \stackrel{\left(a_{0}, \Lambda_{0}\right)}{\longrightarrow} e^{i a_{0} \cdot \hat{a}} \hat{f}_{m^{2}}^{ \pm}\left(\Lambda_{0}^{-1} \hat{a}, \Lambda \Lambda_{0}\right)
\end{array}\right\}
$$

$$
\begin{gathered}
\int|\hat{f}(\hat{a}, \Lambda)|^{2} d \hat{a} d \Lambda=\frac{1}{2} \int_{0}^{\infty} d m^{2}\left[\int\left|\hat{f}_{m^{2}}^{+}(\hat{a}, \Lambda)\right|^{2} d \sigma_{m}^{+}(\hat{a}) d \Lambda+\right. \\
\left.+\int\left|\hat{f}_{m^{2}}^{-}(\hat{a}, \Lambda)\right|^{2} d \sigma_{m}^{-}(\hat{a}) d \Lambda\right]+\frac{1}{2} \int_{-\infty}^{0} d m^{2} \int\left|\hat{f}_{m^{2}}(\hat{a}, \Lambda)\right|^{2} d \sigma_{m}(\hat{a}) d \Lambda
\end{gathered}
$$

where $d \sigma_{m}^{+}(\hat{a}), d \sigma_{m}^{-}(\hat{a}), d \sigma_{m}(\hat{a})$ are invariant measures for $\Omega_{m}^{+}, \Omega_{m}^{-}$and $\Omega_{m}$ respectively. This shows that the representation of $\mathscr{P}$ defined by $(6)$ is a direct integral of representations defined by (8). Our problem will now be resolved if we reduce these simpler representations.

\section{II}

First, we study, the representation corresponding to $\hat{f}_{m^{2}}^{+}(\hat{a}, \Lambda)$, denoted now, in short, by $\varphi(\hat{a}, \Lambda)$. These functions are defined on $\Omega_{m}^{+} \times G$ and have square modulus integrable with respect to invariant measure $d \sigma_{m}^{+}(\hat{a}) d \Lambda$.

We can associate to each $\hat{a} \in \Omega_{m}^{+}$the matrix $\left|\begin{array}{ll}\lambda & 0 \\ \zeta & \lambda^{-1}\end{array}\right|, \lambda>0$, the element in $S L(2, C)$ which transforms the apex $Q_{0}$ of $\Omega_{m}^{+}([1])$ into $\hat{a}$. Now, if $\Lambda=\left|\begin{array}{ll}\alpha & \beta \\ \gamma & \delta\end{array}\right|$, we have:

$$
\left|\begin{array}{ll}
\alpha & \beta \\
\gamma & \delta
\end{array}\right|\left|\begin{array}{ll}
\lambda & 0 \\
\zeta & \lambda^{-1}
\end{array}\right|^{-1}=\left|\begin{array}{ll}
\lambda_{1} & 0 \\
\zeta_{1} & \lambda_{1}^{-1}
\end{array}\right|^{-1}\left|\begin{array}{ll}
u & v \\
-\bar{v} & \bar{u}
\end{array}\right|, \lambda_{1}>0,|u|^{2}+|v|^{2}=1
$$

and we write:

$$
F_{\lambda_{1}, \zeta_{1}}(\lambda, \zeta, \tilde{u})=\varphi(\hat{a}, \Lambda), \tilde{u}=\left|\begin{array}{rr}
u & v \\
-\bar{v} & \bar{u}
\end{array}\right| .
$$

Taking (8) into account, we deduce:

$$
F_{\lambda_{1} \zeta_{1}}(\lambda, \zeta, \tilde{u}) \stackrel{\left(a_{0}, \Lambda_{0}\right)}{\longrightarrow} e^{i a_{0} \cdot \hat{a}} F_{\lambda_{1}, \zeta_{1}}\left(\lambda^{\prime}, \zeta^{\prime}, \tilde{u} \tilde{u}^{\prime}\right)
$$

where $\lambda^{\prime}, \zeta^{\prime}, \tilde{u}^{\prime}$ are defined by:

$$
\left|\begin{array}{ll}
\lambda & 0 \\
\zeta & \lambda-1
\end{array}\right|\left|\begin{array}{ll}
\alpha_{0} & \beta_{0} \\
\gamma_{0} & \delta_{0}
\end{array}\right|=\left|\begin{array}{ll}
u^{\prime} & v^{\prime} \\
-\bar{v}^{\prime} & \bar{u}^{\prime}
\end{array}\right|\left|\begin{array}{ll}
\lambda^{\prime} & 0 \\
\zeta^{\prime} & \lambda^{\prime}-1
\end{array}\right| \text {. }
$$


On the other side, one etablish easily, if $d \sigma_{m}^{+}(\hat{a})=\frac{d^{3} \hat{a}}{\hat{a}_{0}}$ :

$\int|\varphi(\hat{a}, \Lambda)|^{2} d \sigma_{m}^{+}(\hat{a}) d \Lambda=m^{2} \int \frac{d \lambda_{1} d \zeta_{1}}{\lambda_{1}^{3}} \int\left|F_{\lambda_{1} \zeta_{1}}(\lambda, \zeta, \tilde{u})\right|^{2} \frac{d \lambda d \zeta}{\lambda^{3}} d \tilde{u}$

where $d \zeta\left(d \zeta_{1}\right)$ denotes the surface element in the complex plane of $\zeta\left(\zeta_{1}\right)$, and $d \tilde{u}$ is the invariant measure on $S U(2)$. From this result, we deduce that the representation of $\mathscr{P}$, defined by (8) is a direct integral of the representations defined by (12).

Since $F_{\lambda_{1}, \zeta_{1}}(\lambda, \zeta, \tilde{u})$ is of square modulus integrable on $S U(2)$ for almost all $\lambda_{1}, \zeta_{1}, \lambda, \zeta$, we shall write ([2]):

$$
F_{\lambda_{1} \zeta_{1}}(\lambda, \zeta, \tilde{u})=\sum_{s} \sum_{j=-s}^{+s} \sum_{j^{\prime}=-s}^{+s} F_{\lambda_{1}, \zeta_{1} ; j^{\prime}, j}^{s}(\lambda, \zeta) D_{j j^{\prime}}^{s}(\tilde{u})
$$

where $s$ runs over all integers or half-integers and where $D_{j^{\prime}}^{\boldsymbol{j}^{\prime}}(\tilde{u})$ denotes the customary matrix element of the $S U(2)$ representation $D^{s}$. From (12), we associate to each $\left(a_{0}, \Lambda_{0}\right)$ the transformation:

$$
F_{\lambda_{1}, \zeta_{1} ; j^{\prime} j}^{s}(\lambda, \zeta) \stackrel{\left(a_{0}, \Lambda_{0}\right)}{\longrightarrow} e^{i a_{0} \cdot \hat{a}} \sum_{k^{\prime}=-s}^{+s} D_{j^{\prime} k^{\prime}}^{s}\left(\tilde{u}^{\prime}\right) F_{\lambda_{1}, \zeta_{1} ; k^{\prime} j}^{s}\left(\lambda^{\prime}, \zeta^{\prime}\right)
$$

which is one possible form for the unitary irreducible representation of $\mathscr{P}$ with mass $m$ and spin $s$ ([1]). Taking into account orthogonality relations for the $D_{j j^{\prime}}^{s}(\tilde{u})$, we have:

$\int \frac{d \lambda d \zeta}{\lambda^{3}} \int d \tilde{u}\left|F_{\lambda_{1} \zeta_{1}}(\lambda, \zeta, \tilde{u})\right|^{2}=\sum_{s, j} \frac{1}{2 s+1} \sum_{j} \int \frac{d \lambda d \zeta}{\lambda^{3}}\left|F_{\lambda_{1}, \zeta_{1} ; j^{\prime} j}(\lambda, \zeta)\right|^{2}$

and this finishes the reduction into irreducible components for the representation of (8) corresponding to $\hat{f}_{m^{2}}^{+}(\hat{a}, \Lambda)$.

Obviously, we can proceed in the same way for the representation (8) corresponding to $\hat{f}_{m^{2}}(\hat{a}, \Lambda)$. Therefore, we have studied the case $m^{2}>0$ in its entirety.

\section{III}

Now, we consider the case $m^{2}<0$. First, we must notice that for almost all elements $\Lambda=\left|\begin{array}{ll}\alpha & \beta \\ \gamma & \delta\end{array}\right|$, we can write:

$$
\left|\begin{array}{ll}
\alpha & \beta \\
\gamma & \delta
\end{array}\right|=\left.\left|\begin{array}{ll}
a & b \\
\bar{b} & \bar{a}
\end{array}\right|\left|\begin{array}{rr}
0 & 1 \\
-1 & 0
\end{array}\right| \begin{array}{ll}
\varepsilon & 0 \\
\zeta & \lambda-1
\end{array}|,| a\right|^{2}-|b|^{2}=1, \quad \varepsilon=0,1, \quad \lambda>0
$$

which is true for $|\delta|^{2}-|\beta|^{2} \neq 0$, or:

$$
\left|\begin{array}{ll}
\alpha & \beta \\
\gamma & \delta
\end{array}\right|=\left|\begin{array}{ll}
\lambda & 0 \\
\zeta & \lambda-1
\end{array}\right|\left|\begin{array}{rr}
0 & 1 \\
-1 & 0
\end{array}\right|^{\delta}\left|\begin{array}{ll}
a & b \\
\bar{b} & \bar{a}
\end{array}\right|
$$

which is true for $|\alpha|^{2}-|\beta|^{2} \neq 0$ ([6]).

If $Q_{0} \in \Omega_{m}$ has coordinates $(0,0,0, m)$ we can associate to each point $\hat{a} \in \Omega_{m}$, the new coordinates $(\varepsilon, \lambda, \zeta)$ which, from (18), label a right coset 
of $S L(2, C)$ with respect to $S U(1,1)$. Writing now:

$\left|\begin{array}{ll}\alpha & \beta \\ \gamma & \delta\end{array}\right|^{-}\left|\begin{array}{ll}\lambda & 0 \\ \zeta & \lambda^{-1}\end{array}\right|^{-1}\left|\begin{array}{rl}0 & 1 \\ -1 & 0\end{array}\right|^{-\varepsilon}=\left|\begin{array}{ll}\lambda_{1} & 0 \\ \zeta_{1} & \lambda^{-1}\end{array}\right|^{-1}\left|\begin{array}{rl}0 & 1 \\ -1 & 0\end{array}\right|^{-\varepsilon_{1}}\left|\begin{array}{ll}a & b \\ \bar{b} & \bar{a}\end{array}\right|, \varepsilon_{1}=0,1$

we shall define $F_{\left(\varepsilon_{1}, \lambda_{1}, \zeta_{1}\right)}(\varepsilon, \lambda, \zeta, \tilde{a})$ by:

$$
F_{\left(\varepsilon_{1}, \lambda_{1}, \zeta_{1}\right)}(\varepsilon, \lambda, \zeta, \tilde{a})=\varphi(\hat{a}, \Lambda), \quad \Lambda=\left|\begin{array}{ll}
\alpha & \beta \\
\gamma & \delta
\end{array}\right|
$$

where $\tilde{a}$ is the matrix $\left|\begin{array}{cc}a & b \\ \bar{b} & \bar{a}\end{array}\right|$ in the right member of $(20)$ and where $(\varepsilon, \lambda, \zeta)$ corresponds to $\hat{a}$. To simplify, we omit the index $m^{2}$.

Transformation (8) gives:

$$
F_{\left(\varepsilon_{1}, \lambda_{1}, \zeta_{1}\right)}(\varepsilon, \lambda, \zeta, \hat{a}) \stackrel{\left(a_{0}, \Lambda_{0}\right)}{\longrightarrow} e^{i a_{0} \cdot \hat{a}} F_{\left(\varepsilon_{1}, \lambda_{1}, \zeta_{1}\right)}\left(\varepsilon^{\prime}, \lambda^{\prime}, \zeta^{\prime}, \tilde{a} \tilde{a}^{\prime}\right)
$$

where $\varepsilon^{\prime}, \lambda^{\prime}, \zeta^{\prime}, \tilde{a}^{\prime}$ are defined by:

$$
\left|\begin{array}{ll}
\lambda & 0 \\
\zeta & \lambda-1
\end{array}\right|\left|\begin{array}{rl}
0 & 1 \\
-1 & 0
\end{array}\right|^{\varepsilon}\left|\begin{array}{ll}
\alpha_{0} & \beta_{0} \\
\gamma_{0} & \delta_{0}
\end{array}\right|=\left|\begin{array}{ll}
a^{\prime} & b^{\prime} \\
\bar{b}^{\prime} & \bar{a}^{\prime}
\end{array}\right|\left|\begin{array}{rl}
0 & 1 \\
-1 & 0
\end{array}\right| \varepsilon^{\prime}\left|\begin{array}{ll}
\lambda^{\prime} & 0 \\
\zeta^{\prime} & \lambda-1
\end{array}\right|
$$

On the other hand, one established immediately with $d \sigma_{m}(\hat{a})=\frac{d^{3} \hat{a}}{\left|\hat{a}_{0}\right|}$ :

$$
\begin{gathered}
\int \mid \varphi(\hat{a}, \Lambda)^{2} d \sigma_{m}(\hat{a}) d \Lambda=m^{2} \sum_{\varepsilon_{1}=0,1} \int \frac{d \lambda_{1} d \zeta_{1}}{\lambda_{3}} \sum_{\varepsilon=0,1} \int \\
\left|F_{\varepsilon_{1}, \lambda_{1}, \zeta_{1}}(\varepsilon, \lambda, \zeta, \tilde{a})\right|^{2} \frac{d \lambda d \zeta}{\lambda^{3}} d \tilde{a}
\end{gathered}
$$

where $d \tilde{a}$ is Haar measure for $S U(1,1)$. Therefore, the representation of $\mathscr{P}$ defined by (8) is a direct integral of the representations defined by (22).

Now, from (23), $F_{\varepsilon_{1}, \lambda_{1}, \zeta_{1}}(\varepsilon, \lambda, \zeta, \tilde{a})$ has square modulus integrable on $S U(1,1)$ for almost all $\lambda, \zeta, \lambda_{1}, \zeta_{1}$. We can thus write (cf. Appendix for the notations):

$$
\begin{gathered}
F_{\left(\varepsilon_{1}, \lambda_{1}, \zeta_{1}\right)}(\varepsilon, \lambda, \zeta, \tilde{a})=\sum_{\eta=0,1} \sum_{n, m=-\infty}^{+\infty} \int_{0}^{\infty} d \varrho F_{\varepsilon_{1}, \lambda_{1}, \zeta_{1}}^{(m, n)}(\varepsilon, \lambda, \zeta ; \varrho, \eta) \times \\
\times D_{n m}(\tilde{a} ; \varrho, \eta)+\sum_{+,-} \sum_{s=2}^{\infty} \sum_{m, n=0}^{\infty} F_{\varepsilon_{1}, \lambda_{1}, \zeta_{1}}^{ \pm(m, n)}\left(\varepsilon, \lambda, \zeta ; \frac{s}{2}\right) D_{n, m}^{ \pm}\left(\tilde{a} ; \frac{s}{2}\right)
\end{gathered}
$$

and from (2.2) obtain the transformations:

$$
\begin{gathered}
F_{\varepsilon_{1}, \lambda_{1}, \zeta_{1}}^{(m, n)}(\varepsilon, \lambda, \zeta ; \varrho, \eta) \stackrel{\left(a_{0}, \Lambda_{0}\right)}{\longrightarrow} e^{i a_{0} \cdot \hat{a}} \sum_{p=-\infty}^{+\infty} D_{m p}\left(\tilde{a}^{\prime} ; \varrho, \eta\right) \times \\
\times F_{\varepsilon_{1}, \lambda_{1}, \zeta_{1}}^{(p, n)}\left(\varepsilon^{\prime}, \lambda^{\prime}, \zeta^{\prime} ; \varrho, \eta\right) \\
F_{\varepsilon_{1}, \lambda_{1}, \zeta_{1}}^{ \pm(m, n)}\left(\varepsilon, \lambda, \zeta ; \frac{s}{2}\right) \stackrel{\left(a_{0}, \Lambda_{0}\right)}{\longrightarrow} e^{i a_{0} \cdot a} \sum_{p=0}^{\infty} D_{m, p}^{ \pm}\left(\tilde{a}^{\prime} ; \frac{s}{2}\right) F_{\varepsilon_{1}, \lambda_{1}, \zeta_{1}}^{ \pm(p, n)}\left(\varepsilon^{\prime}, \lambda^{\prime}, \zeta^{\prime} ; \frac{s}{2}\right) .
\end{gathered}
$$

Taking account of equation (A.6) in the Appendix, it is obvious that our study of the case $m^{2}<0$ is complete, because, in (24) and (25), we recognize one possible form for the unitary irreducible representation of $\mathscr{P}$ with imaginary mass, induced by the representation $D(\tilde{a}, \varrho, \eta)$ and $D^{ \pm}\left(\tilde{a}, \frac{s}{2}\right)$ of the little group $S U(1,1)$. 
In the following $f(a, \Lambda)$ is an infinitely often differentiable function with compact support. If $T^{+(s, m)}(a, \Lambda)$ denotes the operators of the unitary irreducible representation of $\mathscr{P}$ with mass $m$, spin $s$, corresponding to $\Omega_{m}^{+}$, we consider the operator:

$$
\int d a d \Lambda T^{+(s, m)}(a, \Lambda)^{-1} f(a, \Lambda) .
$$

The $T^{+(s, m)}(a, \Lambda)$ acts on Hilbert space of functions $h_{i}(\hat{a}), \hat{a} \in \Omega_{m}^{+}$, $-s \leqq i \leqq s$, such that:

$$
\sum_{i} \int\left|h_{i}(\hat{a})\right|^{2} d \sigma_{m}^{+}(\hat{a})<\infty .
$$

As transformation law, we have:

$$
h_{i}(\hat{a}) \stackrel{\left(a_{0}, \Lambda_{0}\right)}{\longrightarrow} e^{i a_{0} \cdot a} \sum_{i} D_{i, i^{\prime}}^{s}\left(\tilde{u}^{\prime}\right) h_{i^{\prime}}\left(\hat{a}^{\prime}\right)
$$

where $\tilde{u}^{\prime}$ and $\hat{a}^{\prime}$ are defined by:

$$
\Lambda_{a} \Lambda_{0}=\tilde{u}^{\prime} \Lambda_{\tilde{a}^{\prime}}
$$

Here, $\Lambda_{\hat{a}}$ denotes the matrix $\left|\begin{array}{ll}\lambda & 0 \\ \zeta & \lambda^{-1}\end{array}\right|$ which transforms $Q_{0}$ into $\hat{a}$. We have now:

with

$$
\begin{aligned}
& \int d a d \Lambda f(a, \Lambda) T^{+(s, m)}(a, \Lambda)^{-1} h_{j}(\hat{a}) \\
= & \int d a d \Lambda f(a, \Lambda) e^{-i \Lambda^{-1} a \cdot a} \sum_{j^{\prime}} D_{j j^{\prime}}^{s}\left(u_{1}^{-1}\right) h_{j^{\prime}}\left(\hat{a}_{1}\right)
\end{aligned}
$$

$$
\Lambda_{\hat{a}} \Lambda^{-1}=\tilde{u}_{1}^{-1} \Lambda_{a_{1}} .
$$

If we choose as a new variable in the right member of (27):

and notice that:

$$
\Lambda_{0}=\Lambda \Lambda_{\hat{a}}^{-1}=\Lambda_{\hat{a}_{1}}^{-1} \tilde{u}_{1}
$$

we can write

$$
d \Lambda_{0}=\frac{1}{m^{2}} d \sigma_{m}^{+}\left(\hat{a}_{1}\right) d \tilde{u}_{1}
$$

$$
\begin{aligned}
& \int d a d \Lambda f(a, \Lambda) T^{+(s, m)}(a, \Lambda)^{-1} h_{j}(\hat{a}) \\
= & \sum_{j^{\prime}} \int d \sigma_{m}^{+}\left(\hat{a}_{1}\right)\left[\int d \tilde{u} \frac{1}{m^{2}} \hat{f}_{m_{2}}^{+}\left(\hat{a}, \Lambda_{a_{1}}^{-1} \tilde{u} \Lambda_{\hat{a}}\right) D_{j^{\prime} j}^{s}(\tilde{u})\right] \times h_{j^{\prime}}\left(\hat{a}_{1}\right) \\
= & \sum_{j^{\prime}} \int d \sigma_{m}^{+}\left(\hat{a}_{1}\right) K_{f}^{+{ }^{\left(j j^{\prime}\right)}}\left(\hat{a}, \hat{a}_{1} ; m, s\right) h_{j^{\prime}}\left(\hat{a}_{1}\right) .
\end{aligned}
$$

From orthogonality relations between the $D_{j j^{\prime}}^{s^{\prime}}(\tilde{u})$ and taking into account equations (11), (15), we conclude immediately:

$$
F_{\lambda_{1}, \zeta_{1} ; j, j^{\prime}}^{+s}(\lambda, \zeta)=m^{2}(2 s+1) K_{f}^{+\left(j j^{\prime}\right)}\left(\hat{a}, \hat{a}_{1} ; m, s\right)
$$

and it is easy to prove that $K_{f}^{+}{ }^{\left(j j^{\prime}\right)}\left(\hat{a}, \hat{a}_{1}, m, s\right)$ is the kernel for a HilbertSchmidt operator. 
It is obvious that we can treat in the same way unitary irreducible representations corresponding to $\Omega_{m}^{-}$. We denote by $K_{f}^{-\left(j, j^{\prime}\right)}\left(\hat{a}, \hat{a}_{1} ; m, s\right)$ the corresponding kernel. For the representations with imaginary mass induced by representations $D(\tilde{a} ; \varrho, \eta)$ and $D^{ \pm}\left(\tilde{a} ; \frac{s}{2}\right)$ of the little group, we can apply a similar procedure with the modifications implied by the particular parametrization of $\Omega_{m}$ and the Plancherel measure on $S U(1,1)$. One can repeat word by word the preceding reasoning, as one will easily see if one takes, as point of departure, a form of representations similar to (24) and (25). We always obtain thus kernels for Hilbert-Schmidt operators.

This being said, we wish now to express $f(a, \Lambda)$ in terms of its components. First of all, we have:

$$
\begin{gathered}
f(a, \Lambda)=\frac{1}{2} \frac{1}{(2 \pi)^{4}} \int_{0}^{\infty} d m^{2} \\
{\left[\hat{f}_{m^{2}}^{+}(\hat{a}, \Lambda) e^{i \Lambda^{-1} a \cdot \hat{a}} d \sigma_{m}^{+}(\hat{a})+\int \hat{f}_{m^{2}}^{-}(\hat{a}, \Lambda) e^{i \Lambda^{-1} a \cdot a} d \sigma_{m}^{-}(\hat{a})\right]+} \\
+\frac{1}{2} \frac{1}{(2 \pi)^{4}} \int_{-\infty}^{0} d m^{2} \int \hat{f}_{m^{2}}(\hat{a}, \Lambda) e^{i \Lambda^{-1} a \cdot \hat{a}} d \sigma_{m}(\hat{a}) .
\end{gathered}
$$

We give detailed calculations for the first term in the right member; the other terms can be treated in the same way. We can write:

$$
\begin{aligned}
\int \hat{f}_{m_{2}}^{+}(\hat{a}, \Lambda) e^{i \Lambda^{-1} \hat{a} \cdot a} d \sigma_{m}^{+}(\hat{a}) & =\int d \sigma_{m}^{+}(\hat{a}) \hat{f}_{m}^{+}\left(\hat{a}, \Lambda_{\hat{a}_{1}}^{-1} \tilde{u} \Lambda \hat{a}\right) e^{i\left(\Lambda_{\hat{a}}^{-1} \tilde{u}^{-1} \Lambda_{a_{1}}\right) a \cdot \hat{a}} \\
& =\int d \sigma_{m}^{+}(\hat{a}) \hat{f}_{m^{2}}^{+}\left(\hat{a}, \Lambda_{\hat{a}_{1}}^{-1} \tilde{u} \Lambda_{\hat{a}}\right) e^{i a \cdot \hat{a}_{1}}
\end{aligned}
$$

by the definition of the $\Lambda_{a}$ 's. Then:

$$
\begin{gathered}
\int d \sigma_{m}^{+}(\hat{a}) \hat{f}_{m_{2}}^{+}(\hat{a}, \Lambda) e^{i \Lambda^{-1} a \cdot \hat{a}}=\sum_{s}(2 s+1) \times \\
\times \sum_{j, j^{\prime}} m^{2} \int d \sigma_{m}^{+}(\hat{a}) K_{f}^{+\left(j, j^{\prime}\right)}\left(\hat{a}, \hat{a}_{1} ; m, s\right) D_{j^{\prime} j}^{s}(\tilde{u}) e^{i a \cdot \hat{a}_{1}}
\end{gathered}
$$

and $\Lambda_{a_{1}}, \tilde{u}, \Lambda_{\hat{a}}$ are such that

Now, let us consider:

$$
\Lambda_{\hat{a}_{1}} \cdot \Lambda=\tilde{u} \cdot \Lambda_{\hat{a}} .
$$

We have:

$$
T^{+(s, m)}(a, \Lambda) \int d a^{\prime} d \Lambda^{\prime} f\left(a^{\prime}, \Lambda^{\prime}\right) T^{+(s, m)}\left(a^{\prime}, \Lambda^{\prime}\right)^{-1} .
$$

$$
\begin{aligned}
& T^{+(s, m)}(a, \Lambda) \int d a^{\prime} d \Lambda^{\prime} f\left(a^{\prime}, \Lambda^{\prime}\right) T^{+(s, m)}\left(a^{\prime}, \Lambda^{\prime}\right)^{-1} h_{j}(\hat{a}) \\
= & T^{+(s, m)}(a, \Lambda) \sum_{j^{\prime}} \int d \sigma_{m}^{+}\left(\hat{a}_{1}\right) K_{f}^{+,\left(j, j^{\prime}\right)}\left(\hat{a}, \hat{a}_{1} ; m, s\right) h_{j^{\prime}}\left(\hat{a}_{1}\right) \\
= & e^{i a \cdot \hat{a}} \sum_{k} D_{j k}^{s}\left(\tilde{u}^{\prime}\right) \int d \sigma_{m}^{+}\left(\hat{a}_{1}\right) K_{f}^{+\left(k, j^{\prime}\right)}\left(\hat{a}^{\prime}, \hat{a}_{1} ; m, s\right) h_{j^{\prime}}\left(\hat{a}_{1}\right)
\end{aligned}
$$

where $\tilde{u}^{\prime}, \hat{a}^{\prime}$ are defined by:

$$
\Lambda_{a} \Lambda=\tilde{u}^{\prime} \Lambda_{a^{\prime}}
$$


From this follows:

$$
\begin{aligned}
& \sum_{i, j^{\prime}} \int d \sigma_{m}^{+}(\hat{a}) K_{f}^{+\left(j, j^{\prime}\right)}\left(\hat{a}, \hat{a}_{1} ; m, s\right) D_{j^{\prime} j}^{s}(\tilde{u}) e^{i a \cdot \hat{a}_{1}} \\
= & \operatorname{Tr} T^{+(s, m)}(a, \Lambda) \int d a^{\prime} d \Lambda^{\prime} f\left(a^{\prime}, \Lambda^{\prime}\right) T^{+(s, m)}\left(a^{\prime}, \Lambda^{\prime}\right)^{-1} \\
= & \operatorname{Tr} \int d a^{\prime} d \Lambda^{\prime} f_{(a, \Lambda)}\left(a^{\prime}, \Lambda^{\prime}\right) T^{+(s, m)}\left(a^{\prime}, \Lambda^{\prime}\right)^{-1}
\end{aligned}
$$

where $f_{(a, \Lambda)}\left(a^{\prime}, \Lambda^{\prime}\right)$ is the right-translated by $(a, \Lambda)$ of $f\left(a^{\prime}, \Lambda^{\prime}\right)$ :

$$
f_{(a, \Lambda)}\left(a^{\prime}, \Lambda^{\prime}\right)=f\left(a^{\prime}+\Lambda^{\prime} a, \Lambda^{\prime} \Lambda\right) .
$$

If we denote by $T^{+(s, m)}(f)$ the quantity:

$$
\operatorname{Tr} \int f\left(a^{\prime}, \Lambda^{\prime}\right) T^{+(s, m)}\left(a^{\prime}, \Lambda^{\prime}\right) d a^{\prime} d \Lambda^{\prime}
$$

we can write finally, taking into account the unitary properties:

$$
\int d \sigma_{m}^{+}(\hat{a}) \hat{f}_{m^{2}}^{+}(\hat{a}, \Lambda) e^{i \Lambda^{-1} a \cdot \hat{a}}=m^{2} \sum_{s}(2 s+1) \overline{T^{+(s, m)}\left(f_{(a, \Lambda)}\right)} .
$$

With similar calculations for the other terms in the right member of (30), we obtain (cf. Appendix):

$$
\begin{aligned}
f(a, \Lambda)= & \left.\frac{1}{2} \frac{1}{(2 \pi)^{4}} \Sigma(2 s+1) \int_{0}^{\infty} m^{2} d m^{2} \overline{\left(\overline{T+(s, m)}\left(f_{(a, \Lambda)}\right)\right.}+\overline{T^{-(s, m)}\left(f_{(a, \Lambda)}\right)}\right)+ \\
& +\frac{1}{2} \frac{1}{(2 \pi)^{4}} \int_{-\infty}^{0}\left|m^{2}\right| d m^{2} \int_{0}^{\infty} d \varrho \varrho \operatorname{th} \frac{\pi \varrho}{2} \overline{T^{\varrho, 0, i m}\left(f_{(a, \Lambda)}\right)}+ \\
& +\frac{1}{2} \frac{1}{(2 \pi)^{4}} \int_{-\infty}^{0}\left|m^{2}\right| d m^{2} \int_{0}^{\infty} d \varrho \varrho \operatorname{cth} \frac{\pi \varrho}{2} \overline{T^{\varrho, 1, i m}\left(f_{(a, \Lambda)}\right)}+ \\
& +\frac{1}{2} \frac{1}{(2 \pi)^{4}} \sum_{+,-} \sum_{s=1}^{\infty}(s-1) \int_{-\infty}^{0}\left|m^{2}\right| d m^{2} \overline{T^{ \pm\left(\frac{s}{2}, i m\right)}\left(f_{(a, \Lambda)}\right)}
\end{aligned}
$$

with obvious notations.

In particular, for $a=0, \Lambda=e$ :

$$
\begin{aligned}
f(0, e)= & \frac{1}{2} \frac{1}{(2 \pi)^{4}} \sum_{s}(2 s+1) \sum_{+,-} \int_{0}^{\infty} m^{2} d m^{2} \overline{\left(T^{ \pm(s, m)}(f)\right)}+ \\
& +\frac{1}{2} \frac{1}{(2 \pi)^{4}} \int_{\infty}^{0}|m|^{2} d m^{2} \int_{0}^{\infty} d \varrho \varrho \operatorname{th} \frac{\pi \varrho}{2} \overline{T^{\varrho, 0, i m}(f)}+ \\
& +\frac{1}{2} \frac{1}{(2 \pi)^{4}} \int_{-\infty}^{0}\left|m^{2}\right| d m^{2} \int_{0}^{\infty} d \varrho \varrho \operatorname{cth} \frac{\pi \varrho}{2} \overline{T^{0,1, i m}(f)}+ \\
& +\frac{1}{2} \frac{1}{(2 \pi)^{4}} \sum_{s=1}^{\infty}(s-1) \sum_{+,-} \int_{-\infty}^{0}\left|m^{2}\right| d m^{2} \overline{\left(T^{ \pm\left(\frac{s}{2}, i m\right)}(f)\right)} .
\end{aligned}
$$


Finally, denoting by $K_{f}^{+}(s, m), K_{f}^{-}(s, m), K_{f}(\varrho, \eta, i m)$ and $K_{f}^{ \pm}\left(\frac{s}{2}, i m\right)$ the operator corresponding to kernels connected to representations appearing in (31), (32), we obtain, applying the same calculations to (5) and $(9)$ :

$$
\begin{gathered}
\int|f(a, \Lambda)|^{2} d a d \Lambda \\
=\frac{1}{2} \frac{1}{(2 \pi)^{4}} \sum \sum(2 s+1) \int_{+,-}^{\infty} m^{2} d m^{2} \operatorname{Tr} K_{f}^{ \pm}(s, m) K_{f}^{ \pm}(s, m)^{*}+ \\
+\frac{1}{2} \frac{1}{(2 \pi)^{4}} \int_{-\infty}^{0}\left|m^{2}\right| d m^{2} \int_{0}^{\infty} d \varrho d \varrho \operatorname{th} \frac{\pi \varrho}{2} \operatorname{Tr} K_{f}(\varrho, 0, i m) K_{f}(\varrho, 0, i m)^{*}+ \\
+\frac{1}{2} \frac{1}{(2 \pi)^{4}} \int_{-\infty}^{0}\left|m^{2}\right| d m^{2} \int_{0}^{\infty} d \varrho \varrho \operatorname{cth} \frac{\pi \varrho}{2} \operatorname{Tr} K_{f}(\varrho, 1, i m) K_{f}(\varrho, 1, i m)^{*}+ \\
+\frac{1}{2} \frac{1}{(2 \pi)^{4}} \sum_{+,-} \sum_{s \equiv 1}^{\infty}(s-1) \int_{-\infty}^{0} \mid n d m^{2} \operatorname{Tr} K_{f}^{ \pm}\left(\frac{s}{2}, i m\right) K_{f}^{ \pm}\left(\frac{s}{2}, i m\right) .
\end{gathered}
$$

As the infinitely often differentiable functions with compact support are dense in the Hilbert space of functions with square modulus integrable on $\mathscr{P},(33)$ is still true for all such functions. So, (31) and (33) contain the essential results concerning the Fourier transform on $\mathscr{P}$, this last being understood as in Guelfand's work ([7]).

\section{Appendix}

On unitary representation in principal series of $S U(1,1)$ and Plancherel formula

a) Continuous representations in the principal series. Let $\mathscr{H}$ be the Hilbert space the elements of which are functions $f(\varphi)$ such that:

$$
\int_{0}^{2 \pi}|f(\varphi)|^{2} d \varphi<\infty
$$

Let us associate to each element $\tilde{a}=\left|\begin{array}{cc}a & b \\ \bar{b} & \bar{a}\end{array}\right|$ of $S U(1,1)$ the transformation

$f(\varphi) \rightarrow\left(b e^{i \varphi}+\bar{a}\right)^{i \frac{\varrho}{2}+\frac{\eta}{2}-\frac{1}{2}}\left(\bar{b} e^{-i \varphi}+a\right)^{i \frac{\varrho}{2}-\frac{\eta}{2}-\frac{1}{2}} f\left(\varphi^{\prime}\right)=D(\tilde{a} ; \varrho, \eta) f(\varphi)$

where $\varrho>0, \eta=0$ or 1 and $\varphi^{\prime}$ is given by:

$$
e^{i \varphi^{\prime}}=\frac{a e^{i \varphi}+\bar{b}}{b e^{i \varphi}+\bar{a}} .
$$

(A.1) defines a unitary irreducible representation of $S U(1,1)$ for which Casimir's operator has the value:

$$
q=\frac{1}{4}+\frac{\varrho^{2}}{4} .
$$


If $\eta=0$, one obtains representations, isomorphic to representation $C_{q}^{0}$ in Bargmann's work and, if $\eta=1$, to representations $C_{q}^{1 / 2}\left(q>\frac{1}{4}\right)$.

We take as a norm in $\mathscr{H}$, Bargmann's value:

$$
\|f\|=\left(\frac{1}{2 \pi} \int_{0}^{\infty}|f(\varphi)|^{2} d \varphi\right)^{\frac{1}{2}} .
$$

b) Discrete representation in the principal series. Let $\mathscr{H}_{s}$ be the Hilbert space the elements of which are functions $f(\zeta)$, analytic in the disk $|\zeta|<1$, and such that:

$$
\int_{|\zeta|<1}|f(\zeta)|^{2}\left(1-|\zeta|^{2}\right)^{s-2} d \zeta<\infty \quad s \geqq 2 .
$$

Let us associate to each element $\tilde{a}=\left|\begin{array}{cc}\frac{a}{b} & \bar{a} \\ \bar{a}\end{array}\right|$ of $S U(1,1)$ the transformation

$$
f(\zeta) \rightarrow(b \zeta+\bar{a})^{-s} f\left(\frac{a \zeta+\bar{b}}{b \zeta+\bar{a}}\right)=D^{+}\left(\tilde{a} ; \frac{s}{2}\right) f(\zeta)
$$

or the transformation:

$$
f(\zeta) \rightarrow(\bar{b} \zeta+a)^{-s} f\left(\frac{\bar{a} \zeta+b}{\bar{b} \zeta+a}\right)=D^{-}\left(\tilde{a} ; \frac{s}{2}\right) f(\zeta) .
$$

We define thus unitary irreducible representations isomorphic to representations $D_{s / 2}^{ \pm}$in Bargmann's work.

We take as a norm in $\mathscr{H}_{s}$, Bargmann's value:

$$
\|f\|=\left(\frac{s-1}{\pi} \int_{|\zeta|<1}\left(1-|\zeta|^{2}\right)^{s-2}|f(\zeta)|^{2} d \zeta\right)^{+1 / 2}
$$

The discrepancies between our formulas and those of BARgManN come from the dissimilar action of the group on homogeneous spaces (unit circle, unit disk): in our representation the group acts from the right.

c) Plancherel formula and regular representation ([4], [5]). Let $D_{n, m}(\tilde{a} ; \varrho, \eta)$ be matrix elements of $D(\tilde{a} ; \varrho, \eta)$ in the orthonormal basis $e^{i n \xi},-\infty \leqq n \leqq+\infty$ and let $D_{n, m}^{ \pm}\left(\tilde{a} ; \frac{s}{2}\right)$ be matrix elements of $D^{ \pm}\left(\tilde{a} ; \frac{s}{2}\right)$ in the orthonormal basis $\left(\frac{n !(s-1) !}{(n+s-1) !}\right)^{1 / 2} \zeta^{n}, n=0,1, \ldots$ It result from Bargmann's work that this set of function is a complete system in the Hilbert space whose elements are functions with square modulus integrable on $S U(1,1)$. Let $\phi(\tilde{a})$ be such a function; for almost all $\tilde{a}$, we can write

$$
\begin{aligned}
\phi(\tilde{a})= & \sum_{\eta=0,1} \sum_{n, m=-\infty}^{\infty} \int_{0}^{\infty} d \varrho \phi_{n m}(\varrho, \eta) D_{n m}(\tilde{\alpha} ; \varrho, \eta)+ \\
& +\sum_{+,-} \sum_{s=2}^{\infty} \phi_{n, m}^{ \pm}\left(\frac{s}{2}\right) D_{n, m}^{ \pm}\left(\tilde{a} ; \frac{2}{s}\right) .
\end{aligned}
$$


According to orthogonality relations between matrix elements we have:

Further

$$
\left.\begin{array}{rl}
\varphi_{n m}(\varrho, 0) & =\varrho \operatorname{th} \frac{\pi \varrho}{2} \int d \tilde{a} \phi(\tilde{a}) \overline{D_{n, m}(\tilde{a}, \varrho, 0)} \\
\phi_{n m}(\varrho, 1) & =\varrho \operatorname{cth} \frac{\pi \varrho}{2} \int d \tilde{a} \phi(\tilde{a}) D_{n, m}(\tilde{a} ; \varrho, 1) \\
\phi_{n, m}^{ \pm}\left(\frac{s}{2}\right) & =(s-1) \int d \tilde{a} \phi(\tilde{a}) D_{n, m}^{ \pm}\left(\tilde{a} ; \frac{s}{2}\right) \cdot
\end{array}\right\}
$$

$$
\begin{gathered}
\int|\phi(\tilde{a})|^{2} d \tilde{a}= \\
=\sum_{n, m=\infty}^{+\infty}\left[\int_{0}^{\infty} d \varrho \varrho \operatorname{th} \frac{\pi \varrho}{2}\left|\phi_{n, m}(\varrho, 0)\right|^{2}+\int_{0}^{\infty} d \varrho \varrho \operatorname{cth} \frac{\pi \varrho}{2}\left|\phi_{n, m}(\varrho, 1)\right|^{2}\right]+ \\
+\sum_{s=1}^{\infty}(s-1) \sum_{n, m=0}^{\infty}\left(\left|\phi_{n, m}^{+}\left(\frac{s}{2}\right)\right|^{2}+\left|\phi_{n, m}^{-}\left(\frac{s}{2}\right)\right|^{2}\right) .
\end{gathered}
$$

If we replace $\phi(\tilde{a})$ by its translated $\phi\left(\tilde{a} \tilde{a}_{0}\right)$, the coefficients in A.5 become, taking into account unitarity and invariance of $d \tilde{a}$ :

$$
\sum_{p=-\infty}^{+\infty} D_{m, p}\left(\tilde{a}_{0} ; \varrho, \eta\right) \phi_{n, p}(\varrho, \eta), \sum_{p=0}^{\infty} D_{m, p}^{ \pm}\left(\tilde{a}_{0} ; \frac{s}{2}\right) \phi_{n, p}^{ \pm}\left(\frac{s}{2}\right) .
$$

So, for $n$ fixed, vector functions $\phi_{n, m}(\varrho, \eta), \phi_{n, m}^{ \pm}\left(\frac{s}{2}\right)$ transform according to irreducible representations of $S U(1,1)$ and this with A.6, resolves the problem of decomposing the right regular representation of $S U(1,1)$.

\section{Bibliography}

[1] Rideau, G.: Ann. Inst. Poincaré 3, 339 (1965).

[2] Nammark: Representations linèaires du groupe de Lorentz. Paris: Dunod 1962.

[3] Bargmann: Ann. Math. 48, 568 (1947).

[4] Harish-Chandra: Proc. Nat. Acad. Sci. 38, 337 (1952).

[5] Pukansky: Bull. Am. Math. Soc. 69, 504 (1963).

[6] Graev: Trudy Mosk. Math. Obšč. 7, 335 (1958) (in russian).

[7] GUEFFAND, Graev, and VILENKIN : Obobščenye functsi, tom $V$ (für $f$ ) (in russian). 